\title{
Pose estimation by extended Kalman filter using noise covariance matrices based on sensor output
}

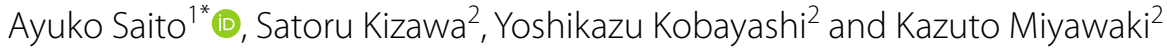

\begin{abstract}
This paper presents an extended Kalman filter for pose estimation using noise covariance matrices based on sensor output. Compact and lightweight nine-axis motion sensors are used for motion analysis in widely various fields such as medical welfare and sports. A nine-axis motion sensor includes a three-axis gyroscope, a three-axis accelerometer, and a three-axis magnetometer. Information obtained from the three sensors is useful for estimating joint angles using the Kalman filter. The extended Kalman filter is used widely for state estimation because it can estimate the status with a small computational load. However, determining the process and observation noise covariance matrices in the extended Kalman filter is complicated. The noise covariance matrices in the extended Kalman filter were found for this study based on the sensor output. Postural change appears in the gyroscope output because the rotational motion of the joints produces human movement. Therefore, the process noise covariance matrix was determined based on the gyroscope output. An observation noise covariance matrix was determined based on the accelerometer and magnetometer output because the two sensors' outputs were used as observation values. During a laboratory experiment, the lower limb joint angles of three participants were measured using an optical 3D motion analysis system and nine-axis motion sensors while participants were walking. The lower limb joint angles estimated using the extended Kalman filter with noise covariance matrices based on sensor output were generally consistent with results obtained from the optical 3D motion analysis system. Furthermore, the lower limb joint angles were measured using nine-axis motion sensors while participants were running in place for about $100 \mathrm{~s}$. The experiment results demonstrated the effectiveness of the proposed method for human pose estimation.
\end{abstract}

Keywords: Kalman filter, Motion sensor, Noise covariance matrix, Pose estimation, Sensor fusion

\section{Introduction}

Compact and lightweight nine-axis motion sensors have been developed through advances in micro-electromechanical systems technology; they have come to be used for motion analysis in widely various fields [1-8]. The nine-axis motion sensors are applicable both indoors and outdoors because of their portability. Several experiments have been conducted to measure the motion of a skier gliding down a slope and jumping off a hill using

\footnotetext{
*Correspondence: saito@cc.kogakuin.ac.jp

1 Department of Mechanical Science and Engineering, Kogakuin University, 2665-1 Nakanomachi, Hachioji, Tokyo 192-0015, Japan Full list of author information is available at the end of the article
}

motion sensors $[9,10]$. The nine-axis motion sensors include a three-axis gyroscope, a three-axis accelerometer, and a three-axis magnetometer. Using information obtained from the motion sensors, several sensor fusion algorithms have been proposed for pose estimation: as one example, a sensor fusion algorithm that can correct gyroscope drift using information obtained from the other two sensors has been used for human pose estimation during daily activities and exercise [11-13]. Furthermore, a sensor fusion algorithm able to correct the magnetometer output using information obtained from a gyroscope has been used for pose estimation in a variable magnetic field $[14,15]$. The Kalman filter [16-20] and the 
complementary filter [21-25] are some pose estimation methods using sensor fusion.

The Kalman filter estimates the system state with a small computational load. Nevertheless, determining the process and observation noise covariance matrices in the Kalman filter is complicated. For a case in which the process and observation noise covariance matrices are timeinvariant, the estimation accuracy might decrease if the sensor output noise increases. Moreover, the noise of the sensor output might vary because of long-term measurements. For that reason, adjusting the noise covariance matrices based on sensor output is important.

To estimate the lower limb joint angles for this study, a method was devised to determine the process and observation noise covariance matrices in the extended Kalman filter based on sensor output. The postural change appears in the gyroscope output because the rotational motion of the joints produces human movement. Therefore, the process noise covariance matrix was set based on the gyroscope output. When the accelerometer output increased, the observation noise covariance matrix was set to increase. The observation noise covariance matrix was also set to increase when the magnetometer output drastically changed. During a laboratory experiment, the lower limb joint angles of three participants were measured using an optical 3D motion analysis system and nine-axis motion sensors while the participants were walking. Several studies have demonstrated that an optical 3D motion analysis system measured human movement with high accuracy. Therefore, the system is used for verifying the pose estimation accuracy in widely diverse fields [26-29]. We verified the accuracy of the proposed method by comparing its results to those of an optical 3D motion analysis system. Furthermore, the lower limb joint angles were measured using nine-axis motion sensors while the participants were running in place. Finally, the effectiveness of the proposed method was verified using experiment results.

\section{Measurement method}

\section{Definition of roll-pitch-yaw}

The 3D posture of the sensor is represented by the roll angle $(\phi)$ around the $\mathrm{x}$-axis, the pitch angle $(\theta)$ around the $y$-axis, and the yaw angle $(\psi)$ around the $\mathrm{z}$-axis. The reference coordinate system is a right-handed system with a vertical $\mathrm{z}$-axis. The counterclockwise rotation is defined as positive. The reference coordinate system and the definition of the joint angles are presented in Fig. 1.

\section{Roll-pitch-yaw calculation}

For this study, Euler angles (roll, pitch, and yaw) were calculated using nine-axis motion sensors. The

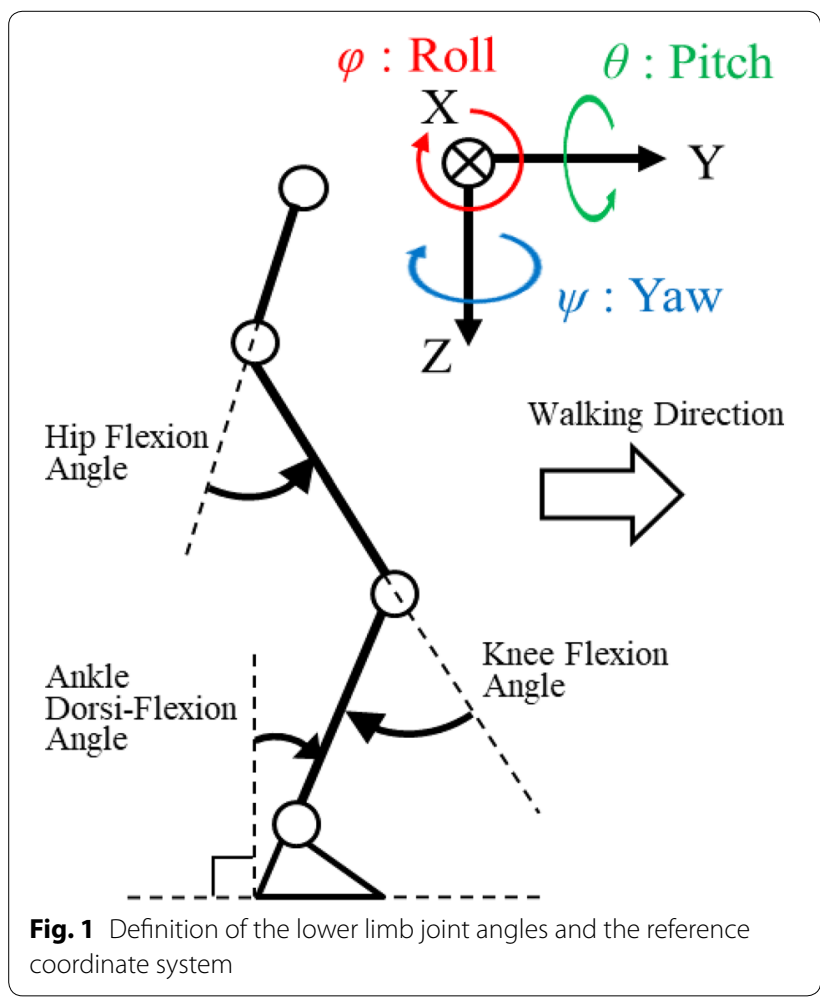

nine-axis motion sensor (SS-WS1792; Sports Sensing Co., Ltd.) used for this study includes a three-axis gyroscope $( \pm 1500 \mathrm{dps})$, a three-axis accelerometer $( \pm 16$ $\mathrm{G})$, and a three-axis magnetometer ( \pm 10 Gauss). The $38 \times 53 \times 11 \mathrm{~mm}$ sensor weighs $30 \mathrm{~g}$.

The initial roll and pitch angles were calculated using the accelerometer output at rest [30,31]. The relation between the acceleration sensor output and the gravitational acceleration in the reference coordinate system is expressed using Eq. (1) because the accelerometer measures only the. gravitational acceleration while at rest:

$$
{ }^{i} A=\left({ }^{O} R_{i}\right)^{T O} A,(i=1,2,3,4)
$$

where.

$$
{ }^{i} A=\left[\begin{array}{l}
{ }^{i} A_{x} \\
{ }^{i} A_{y} \\
{ }^{i} A_{z}
\end{array}\right],{ }^{o} A=\left[\begin{array}{l}
0 \\
0 \\
g
\end{array}\right],
$$

Therein, ${ }^{i} A$ denotes the accelerometer output, ${ }^{\circ} A$ represents the acceleration in the reference coordinate system, and $g$ stands for gravitational acceleration. For the experiment, sensors $1,2,3$, and 4 were placed respectively on the waist, left thigh, left shank, and left foot. In addition, the rotational matrix from the sensor coordinate system to the reference system ${ }^{\circ} R_{i}$ is the following: 


$$
{ }^{o} R_{i}=\left[\begin{array}{ccc}
\cos ^{i} \psi & -\sin ^{i} \psi & 0 \\
\sin ^{i} \psi & \cos ^{i} \psi & 0 \\
0 & 0 & 1
\end{array}\right]\left[\begin{array}{ccc}
\cos ^{i} \theta & 0 & \sin ^{i} \theta \\
0 & 1 & 0 \\
-\sin ^{i} \theta & 0 & \cos ^{i} \theta
\end{array}\right]\left[\begin{array}{ccc}
1 & 0 & 0 \\
0 & \cos ^{i} \phi & -\sin ^{i} \phi \\
0 & \sin ^{i} \phi & \cos ^{i} \phi
\end{array}\right]
$$

Then, the accelerometer output ${ }^{i} A$ is represented by substituting Eq. (2) into Eq. (1) as shown below:

$$
\left[\begin{array}{l}
{ }^{i} A_{x} \\
{ }^{i} A_{y} \\
{ }^{i} A_{z}
\end{array}\right]=\left[\begin{array}{c}
-\sin ^{i} \theta \cdot g \\
\cos ^{i} \theta \sin i \phi \cdot g \\
\cos ^{i} \theta \cos ^{i} \phi \cdot g
\end{array}\right]
$$

The initial roll and pitch angles using Eq. (3) are:

$$
\begin{aligned}
& { }^{i} \phi_{A}=\operatorname{atan} 2 \frac{i A_{y}}{{ }^{i} A_{z}}\left(-\pi<{ }^{i} \phi_{A}<\pi\right) \\
& { }^{i} \theta_{A}=\operatorname{atan} 2 \frac{-{ }^{i} A_{x}}{\sqrt{{ }^{i} A_{y}^{2}+{ }^{i} A_{z}^{2}}}\left(-\pi<{ }^{i} \theta_{A}<\pi\right)
\end{aligned}
$$

where ${ }^{i} A_{x}{ }^{i} A_{y}$, and ${ }^{i} A_{z}$ respectively denote the accelerometer output for the $x, y$, and $z$ axes. Therein, ${ }^{i} \phi_{A}$ and ${ }^{i} \theta_{A}$ respectively denote the initial roll and pitch.

To correct the yaw angle, calculations require the roll ${ }^{i} \phi_{A}$, pitch ${ }^{i} \theta_{A}$, and magnetometer output as:

$$
\left[\begin{array}{l}
c, i \\
c, i \\
c, i \\
{ }^{c} m_{y} \\
c, i \\
m_{z}
\end{array}\right]=\left[\begin{array}{ccc}
\cos ^{i} \theta_{A} & \sin ^{i} \phi_{A} \sin ^{i} \theta_{A} & \cos ^{i} \phi_{A} \sin ^{i} \theta_{A} \\
0 & \cos ^{i} \phi_{A} & -\sin ^{i} \phi_{A} \\
-\sin { }^{i} \theta_{A} & \sin ^{i} \phi_{A} \cos { }^{i} \theta_{A} & \cos { }^{i} \phi_{A} \cos { }^{i} \theta_{A}
\end{array}\right] \cdot\left[\begin{array}{l}
{ }^{i} m_{x} \\
{ }^{i} m_{y} \\
{ }^{i} m_{z}
\end{array}\right]
$$

where ${ }^{i} m_{x},{ }^{i} m_{y}$, and ${ }^{i} m_{z}$ respectively denote the magnetometer outputs for the $x, y$, and $z$ axes. Therein, ${ }^{c, i} m_{x},{ }^{c, i} m_{y}$, and ${ }^{c, i} m_{z}$ respectively represent the corrected magnetic field data for the $x, y$, and $z$ axes. The positive directions of ${ }^{c, i} m_{x},{ }^{c, i} m_{y}$, and ${ }^{c, i} m_{z}$ coincide with those of each axis of the reference coordinate system in Fig. 1. The $x$-axis pointed in the direction of the azimuth at 112.5 degrees (east-southeast). The $y$-axis pointed in the direction at the azimuth of 202.5 degrees (south-southwest) in the reference coordinates.

The following equation is used for calculating yaw:

$$
{ }^{i} \psi_{m}=\operatorname{atan} 2 \frac{{ }^{c, i} m_{y}}{{ }^{c, i} m_{x}}\left(-\pi<{ }^{i} \psi_{m}<\pi\right)
$$

where ${ }^{i} \psi_{m}$ denotes the azimuth on the $x-y$ plane of the reference coordinates in Fig. 1.
The differential Euler angles in the reference coordinate system are the following:

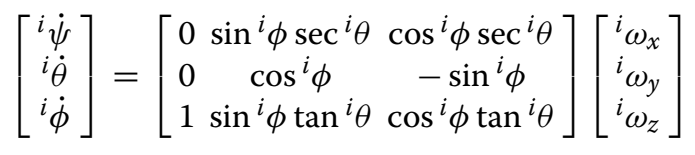

Therein, ${ }^{i} \dot{\phi},{ }^{i} \dot{\theta}$, and ${ }^{i} \dot{\psi}$ respectively represent the differential roll, pitch, and yaw angles, ${ }^{i} \omega_{y}$ and ${ }^{i} \omega_{z}$ respectively stand for the gyroscope output for the $x, y$, and $z$ axes. Then the roll, pitch, and yaw angles are calculated by substituting Eq. (8) into Eq. (9):

$$
\left[\begin{array}{c}
{ }^{i} \psi \\
{ }^{i} \theta \\
{ }^{i} \phi
\end{array}\right]_{t+1}=\int\left[\begin{array}{c}
{ }^{i} \dot{\psi} \\
{ }^{i} \dot{\theta} \\
{ }^{i} \dot{\phi}
\end{array}\right] d t+\left[\begin{array}{c}
{ }^{i} \psi \\
{ }^{i} \theta \\
{ }^{i} \phi
\end{array}\right]_{t}
$$

\section{Extended Kalman filter}

\section{State-space model}

The roll, pitch, and yaw angles of each sensor placed on the lower limb are estimated by the sensor fusion using

the extended Kalman filter. The nonlinear state equation was developed using Eq. (9). The nonlinear observation equation was developed using Eq. (7) and the acceleration sensor output. The nonlinear state and observation equations are shown respectively in Eqs. 10 and 11:

$$
\begin{aligned}
& { }^{i} x_{t+1}={ }^{i} F\left({ }^{i} x_{t},{ }^{i} \omega_{t}\right)+{ }^{i} w_{t}, \\
& { }^{i} y_{t}={ }^{i} H\left({ }^{i} x_{t}\right)+{ }^{i} v_{t},
\end{aligned}
$$

where,

$$
{ }^{i} x_{t}=\left[\begin{array}{c}
{ }^{i} \psi \\
{ }^{i} \theta \\
{ }^{i} \phi
\end{array}\right]_{t}
$$

$$
{ }^{i} F\left(x_{t}, \omega_{t}\right)=\left[\begin{array}{l}
{ }^{i} \psi_{t}+\sin { }^{i} \phi_{t} \sec ^{i} \theta_{t}{ }^{i} \omega_{y, t} \cdot T s+\cos { }^{i} \phi_{t} \sec { }^{i} \theta_{t}{ }^{i} \omega_{z, t} \cdot T s \\
{ }^{i} \theta_{t}+\cos { }^{i} \phi_{t}{ }^{i} \omega_{y, t} \cdot T s-\sin { }^{i} \phi_{t}{ }^{i} \omega_{z, t} \cdot T s \\
{ }^{i} \phi_{t}+{ }^{i} \omega_{x, t} \cdot T s+\sin { }^{i} \phi_{t} \tan { }^{i} \theta_{t}{ }^{i} \omega_{y, t} \cdot T s+\cos { }^{i} \phi_{t} \tan { }^{i} \theta_{t}{ }^{i} \omega_{z, t} \cdot T s
\end{array}\right]
$$




$$
{ }^{i} y_{t}=\left[\begin{array}{c}
{ }^{i} \psi_{m} \\
{ }^{i} A_{S_{x}} \\
{ }^{i} A_{S_{y}} \\
{ }^{i} A_{S_{z}}
\end{array}\right]_{t},{ }_{t}^{i} H\left(x_{t}\right)=\left[\begin{array}{c}
{ }^{i} \psi_{t} \\
\left({ }^{0} R_{i}\right)_{t}^{T}\left[\begin{array}{l}
0 \\
0 \\
g
\end{array}\right]
\end{array}\right]
$$

In those equations, ${ }_{t},{ }^{i} \theta_{t}$, and ${ }^{i} \psi_{t}$ respectively denote Euler angles of the sensor placed on each lower limb segment, as estimated using the extended Kalman filter. $T s$ stands for the sampling time. In addition, ${ }^{i} \omega_{x, t},{ }^{i} \omega_{v, t}$, and ${ }^{i} \omega_{z, t}$ respectively denote the gyroscope outputs for the $x, y$, and $z$ axes. Also, ${ }^{i} A_{S_{x}},{ }^{i} A_{S_{y}}$, and ${ }^{i} A_{S_{z}}$ respectively express the accelerometer output for the $x, y$, and $z$ axes. Therein, ${ }^{i} w_{t}$ and ${ }^{i} v_{t}$ denote white noise.

Yaw angle ${ }^{i} \psi_{m}$, which was calculated using the magnetometer output, and the accelerometer output were used as the observation values in Eq. (11). Equation (1) represents the relation between the accelerometer output and gravitational acceleration. Consequently, the right side of Eq. (1) was used in ${ }^{i} H\left({ }^{i} x_{t}\right)$ of the observation equation. Although the proportion of the centrifugal acceleration and the tangential acceleration in the accelerometer output would have increased during exercise, these acceleration components were processed as observation noise. In addition, ${ }^{i} \psi_{m}={ }^{i} \psi_{t}$ is a simple equation representing the relation between the magnetometer output and the yaw angle of the state values. Therefore, the yaw angle ${ }^{i} \psi_{t}$ of the state value was used in ${ }^{i} H\left({ }^{i} x_{t}\right)$ of the observation equation.

The partial derivatives of ${ }^{i} F\left({ }^{i} x_{t},{ }^{i} \omega_{t}\right)$ and ${ }^{i} H\left({ }^{i} x_{t}\right)$ are shown below:

$$
\begin{aligned}
& { }^{i} f\left({ }^{i} x_{t},{ }^{i} \omega_{t}\right)=\frac{\partial^{i} F\left({ }^{i} x_{t},{ }^{i} \omega_{t}\right)}{\partial^{i} x_{t}}, \\
& { }^{i} h\left({ }^{i} x_{t}\right)=\frac{\partial^{i} H\left({ }^{i} x_{t}\right)}{\partial^{i} x_{t}},
\end{aligned}
$$

Then, the prediction step [Eqs. (14) and (15)] and the filtering step [Eqs. (18), (19), (20)] are calculated using the nonlinear discrete-time system represented by Eqs. (10) and (11)] . Here, Eq. (16) and Eq. (17) are used for calculating the likelihood of the state-space model:

$$
\begin{aligned}
& { }^{i} x_{t+1}^{-}={ }^{i} F\left({ }^{i} x_{t},{ }^{i} \omega_{t}\right) \\
& { }^{i} P_{t+1}^{-}={ }^{i} f_{t}{ }^{i} P_{t}{ }^{i} f_{t}^{T}+{ }^{i} Q_{t}, \\
& { }^{i} V_{t+1}={ }^{i} y_{t+1}-{ }^{i} H\left({ }^{i} x_{t+1}^{-}\right), \\
& { }^{i} B_{t+1}={ }^{i} h_{t+1}{ }^{i} P_{t+1}^{-}{ }^{i} h_{t+1}^{T}+{ }^{i} R_{t},
\end{aligned}
$$

$$
\begin{aligned}
& { }^{i} K_{t+1}={ }^{i} P_{t+1}^{-}{ }^{i} h_{t+1}^{T}\left({ }^{i} h_{t+1}{ }^{i} P_{t+1}^{-}{ }^{i} h_{t+1}^{T}+{ }^{i} R_{t}\right)^{-1}, \\
& { }^{i} x_{t+1}={ }^{i} x_{t+1}^{-}+{ }^{i} K_{t+1}\left({ }^{i} y_{t+1}-{ }^{i} H\left(x_{t+1}^{-}\right)\right) \\
& { }^{i} P_{t+1}=\left(I-{ }^{i} K_{t+1}{ }^{i} h_{t+1}\right){ }^{i} P_{t+1}^{-}
\end{aligned}
$$

In those equations, ${ }^{i} P$ represents the error covariance matrix, ${ }^{i} V$ denotes the prediction error matrix, ${ }^{i} B$ stands for the prediction error variance matrix, and ${ }^{i} K$ denotes the Kalman gain. Therein, ${ }^{i} Q$ and ${ }^{i} R$ respectively denote the covariance matrices of process noise ${ }^{i} w_{t}$ in the nonlinear state equation and observation noise ${ }^{i} v_{t}$ in the nonlinear observation equation.

\section{Noise covariance matrices based on sensor output}

The process and observation noise covariance matrices in the extended Kalman filter were determined based on the state-space model dynamics and the sensor noise. The postural change appears in the gyroscope output because the rotational motion of the joints produces human movement. Consequently, the process noise covariance matrix was determined based on the gyroscope output as presented below:

$$
{ }^{i} Q_{t}=\left[\begin{array}{ccc}
{ }^{i} \Omega_{\omega, t} & 0 & 0 \\
0 & { }^{i} \Omega_{\omega, t} & 0 \\
0 & 0 & { }^{i} \Omega_{\omega, t}
\end{array}\right]
$$

where,

$$
{ }^{i} \Omega_{\omega, t}=a \sqrt{{ }^{i} \omega_{x, t}^{2}+{ }^{i} \omega_{y, t}^{2}+{ }^{i} \omega_{z, t}^{2}}+b
$$

In those expressions, ${ }^{i} \omega_{x, t},{ }^{i} \omega_{y, t}$, and ${ }^{i} \omega_{z, t}$ respectively stand for the gyroscope output for $x, y$, and $z$ axes. Also $a$ and $b$ are adjusting parameters. For this study, $a$ and $b$ were determined to maximize the log-likelihood $\left({ }^{i} L L\right)$ shown in Eq. (22):

$$
{ }^{i} L L=-\frac{N}{2} \ln (2 \pi)-\frac{1}{2} \sum_{j=1}^{N}\left(\ln \left({ }^{i} B_{j}\right)+\frac{{ }^{i} V_{j}^{2}}{{ }^{i} B_{j}}\right)
$$

In that equation, $N$ stands for the number of timeseries data; $j$ represents time-series. In addition, ${ }^{i} B_{j}$ expresses the prediction error variance; ${ }^{i} V_{j}$ signifies the prediction error.

The observation noise covariance matrices must be set at a high value when the sensor noise increases [32]. Therefore, the observation noise covariance matrix was determined based on the accelerometer and magnetometer output because the two sensor outputs were used as 
observation values [33]. The observation noise covariance matrix is presented below:

$$
{ }^{i} R_{t}=\left[\begin{array}{cccc}
{ }^{i} \Omega_{m, t} & 0 & 0 & 0 \\
0 & { }^{i} \Omega_{a, t} & 0 & 0 \\
0 & 0 & { }^{i} \Omega_{a, t} & 0 \\
0 & 0 & 0 & { }^{i} \Omega_{a, t}
\end{array}\right]
$$

where,

$$
\begin{aligned}
& { }^{i} \Omega_{m, t}=c\left(\sqrt{{ }^{c, i} m_{x, t}^{2}+{ }^{c, i} m_{y, t}^{2}+{ }^{c, i} m_{z, t}^{2}}-\bar{m}\right)+d \\
& { }^{i} \Omega_{a, t}=e\left(\sqrt{{ }^{i} A_{x, t}^{2}+{ }^{i} A_{y, t}^{2}+\left({ }^{i} A_{z, t}-g\right)^{2}}\right)+f
\end{aligned}
$$

In the matrix, ${ }^{c, i} m_{x, t},{ }^{c, i} m_{y, t}$, and ${ }^{c, i} m_{z, t}$ respectively denote corrected magnetic field data for the $x, y$, and $z$ axes. In addition, $\bar{m}$ represents the average value of the magnetometer output over the entire measurement time. Furthermore, ${ }^{i} A_{x, t},{ }^{i} A_{y, t}$, and ${ }^{i} A_{z, t}$ respectively express the accelerometer outputs for the $x, y$, and $z$ axes. Therein, $c, d, e$, and $f$ are adjusting parameters. In addition, $c, d$, $e$, and $f$ were determined to maximize the log-likelihood (LL) shown in Eq. (22). Several studies have proposed process and observation noise covariance matrices based on sensor output $[31,33]$. In those earlier studies, noise covariance matrices were produced after calculating the deviation of each sensor using many equations. The adjusting parameters $a$ to $f$ in the noise covariance matrices are determined simply using only log-likelihood calculations presented in Eq. (22). In addition, interaction between the process noise covariance and observation noise covariance is considered in log-likelihood calculations.

The roll, pitch, and yaw angles in the sensor $i$ coordinate system obtained from the sensor fusion are converted into the rotational matrix of the reference coordinate system using Eq. (2). The lower limb joint angles are calculated by substituting Eq. (2) into Eq. (24) as shown below:

$$
{ }^{i-1} R_{i}=\left({ }^{0} R_{i-1}\right)^{T} \cdot\left({ }^{0} R_{i}\right)
$$

Table 1 Anthropometric data

\begin{tabular}{llll}
\hline Participant & Height $[\mathrm{m}]$ & Weight $[\mathrm{kg}]$ & Age [years] \\
\hline A & 1.78 & 60 & 20 \\
B & 1.72 & 65 & 20 \\
C & 1.80 & 56 & 21 \\
\hline
\end{tabular}

In that equation, ${ }^{i-1} R_{i}$ denotes the rotational matrix from the sensor $i$ coordinate system to the sensor $i-1$ system. The hip joint angle is estimated using the output of the two sensors placed on the waist and thigh. The knee joint angle is estimated using the output of the two sensors placed on the thigh and shank. The ankle joint angle is estimated using the output of the two sensors positioned on the shank and foot.

\section{Verification experiment \\ Participants and experiment conditions}

Three healthy participants (A, B, and C) were examined during the experiment. Anthropometric data are shown in Table 1. After maintaining the upright posture for about $5 \mathrm{~s}$, the first step that a participant took was with the left foot. They were instructed to walk using a natural stride in time with a metronome $(70 \mathrm{bpm})$. Measurement started simultaneously when a participant started to maintain the upright posture. The measurements finished when the participant placed the right foot flat on the floor during the sixth step. Following an explanation of the purpose and requirements of the study, the participants gave their written informed consent to participate in the study. Study approval was obtained from the Research Ethics Board, Kogakuin University, and National Institute of Technology, Akita College.

During the experiment, kinematic data were collected using an optical 3D motion analysis system (Bonita 10;

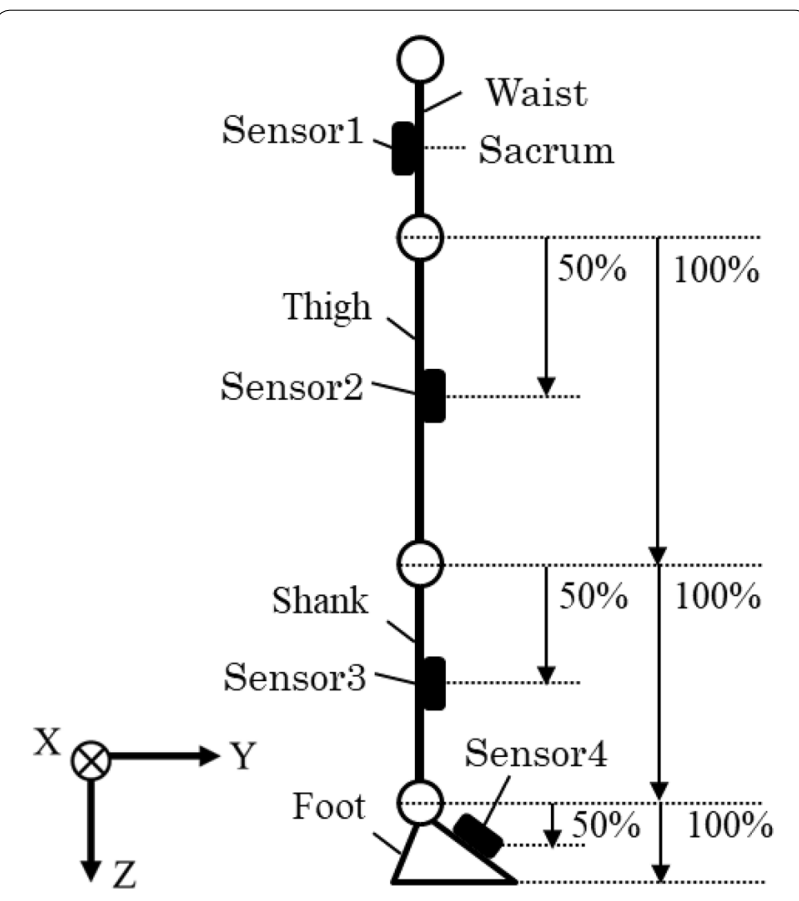

Fig. 2 Sensor positions and sensor coordinate system 
Vicon Motion Systems Ltd.), two force plates (9286; Kistler Japan Co. Ltd.), and four nine-axis motion sensors in synchronization. The heel strike and toe off were ascertained from force plate data. The sensors were placed on the waist, left thigh, left shank, and left foot using double-sided tape and elastic straps. The sensor positions are presented in Fig. 2. Definitions of the length of the thigh, shank, and foot were referred from reports of earlier research [34]. Positions of reflective markers for the optical 3D motion analysis system were found by reference to the Vicon Plug-in Gait model. The sampling frequencies of the nine-axis motion sensors, the optical 3D motion analysis system, and the force plates were $100 \mathrm{~Hz}$.

\section{Results}

Table 2 shows parameters $a$ to $f$ in the walking experiment, which were found to maximize the log-likelihood in Eq. (22). In the walking experiment, more or less equivalent parameters were obtained for all participants.

The joint angles of participant A are depicted in Fig. 3. Black solid curves represent results obtained from the optical 3D motion analysis system. Red solid curves represent results obtained from the extended Kalman filter using the noise covariance matrices based on sensor output, hereinafter designated as NBS. Orange solid curves represent results obtained from NBS, which used gyroscope output for the process noise covariance matrix and which used a constant value for the observation noise covariance matrix, hereinafter designated as NBS (Only process noise). Green solid curves represent results obtained from NBS, which used the constant value for the process noise covariance matrix and which used accelerometer and magnetometer output for the observation noise covariance matrix, hereinafter designated as NBS (Only observation noise). Blue solid curves present results obtained from the extended Kalman filter using the constant noise covariance, hereinafter designated as CNC. In addition, $\Omega \omega(=0.0005), \Omega \mathrm{m}(=1500)$, and $\Omega \mathrm{a}$ $(=1500)$ of $\mathrm{CNC}$ were determined to maximize the log likelihood (LL) shown in Eq. (22). The horizontal axis shows the normalized time, where one gait cycle is $100 \%$. One gait cycle in Fig. 3 extends from the beginning of the stance phase of the left leg (the third step) until the end of the swing phase. Table 3 shows root mean square errors (RMSE) between the estimated results and the results obtained from the 3D motion analysis system.

Joint angles obtained from the three NBSs and CNC are generally consistent with the results obtained using the optical 3D motion analysis system. Results show the same tendency for joint angle variation as that found in an earlier study [35].

The ankle joint angle obtained from NBS (Only process noise) is generally consistent with results obtained using the optical 3D motion analysis system at the dorsiflexion peak after toe-off. The ankle joint angle obtained

Table 2 Adjusting parameters of NBS in the walking experiment

\begin{tabular}{|c|c|c|c|c|c|c|}
\hline \multicolumn{7}{|c|}{ (a) Ankle joint } \\
\hline \multirow[t]{2}{*}{ Participant } & \multicolumn{6}{|c|}{ Adjusting parameters } \\
\hline & $a$ & $b$ & c & $d$ & $e$ & $\bar{f}$ \\
\hline A & 0.00001 & 0 & 0.1 & 0 & 1 & 0 \\
\hline B & 0.00001 & 0 & 0.1 & 0 & 1 & 0 \\
\hline C & 0.00001 & 0 & 0.1 & 0 & 1 & 0 \\
\hline \multicolumn{7}{|c|}{ (b) Knee joint } \\
\hline \multirow[t]{2}{*}{ Participant } & \multicolumn{6}{|c|}{ Adjusting parameters } \\
\hline & $\bar{a}$ & $b$ & c & $d$ & $e$ & $f$ \\
\hline A & 0.00001 & 0 & 0.1 & 0 & 1 & 0 \\
\hline B & 0.00001 & 0 & 0.1 & 0 & 1 & 0 \\
\hline C & 0.00001 & 0 & 0.1 & 0 & 10 & 0 \\
\hline \multicolumn{7}{|l|}{ (c) Hip joint } \\
\hline \multirow[t]{2}{*}{ Participant } & \multicolumn{6}{|c|}{ Adjusting parameters } \\
\hline & $a$ & $b$ & $c$ & $d$ & $e$ & $f$ \\
\hline A & 0.00001 & 0 & 0.1 & 0 & 1 & 0 \\
\hline B & 0.00001 & 0 & 0.1 & 0 & 1 & 0 \\
\hline C & 0.00001 & 0 & 0.1 & 0 & 10 & 0 \\
\hline
\end{tabular}




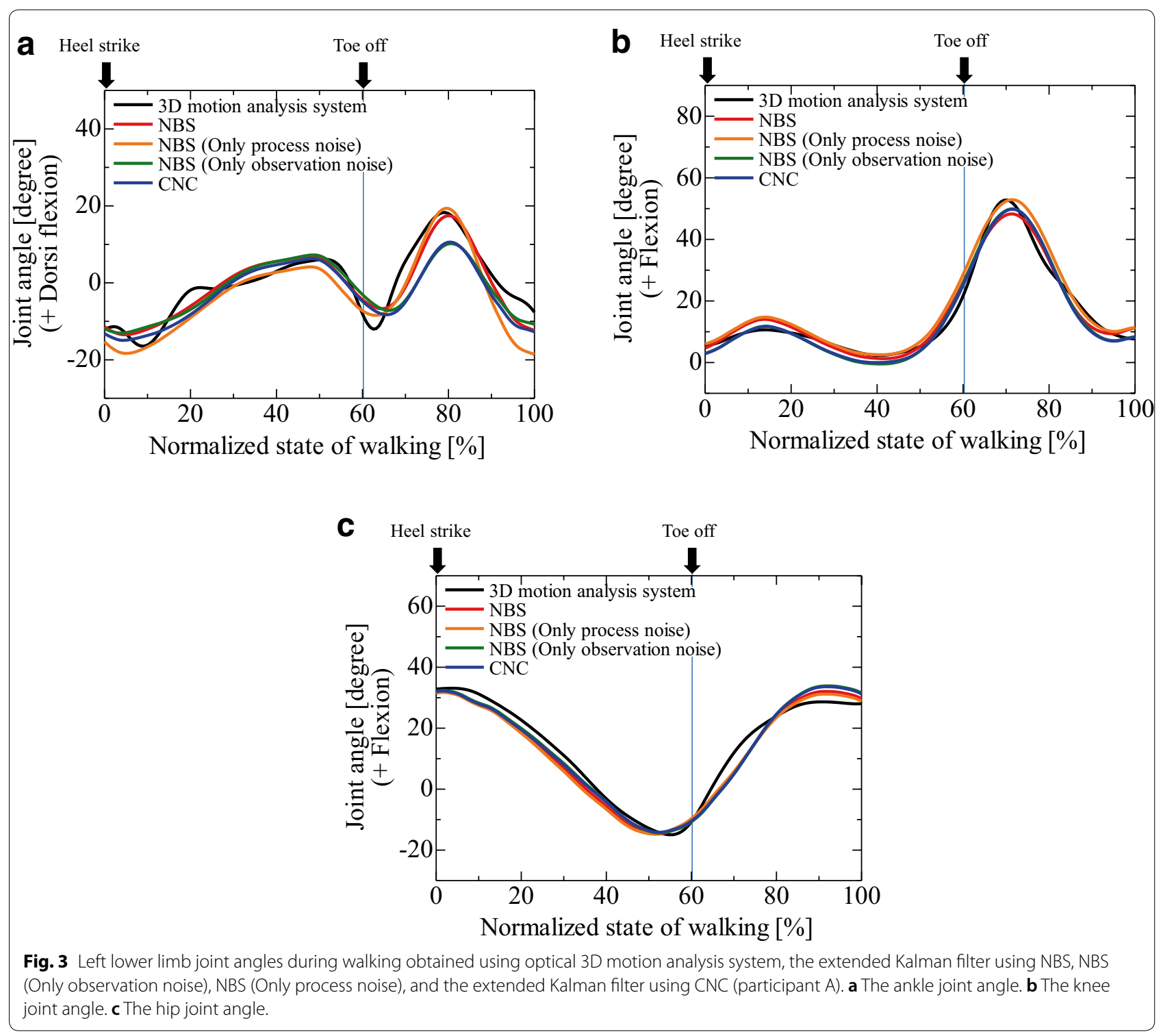

Table 3 Root mean square errors for results obtained from two extended Kalman filters and 3D motion analysis system results (participant $A$ )

\begin{tabular}{llll}
\hline Noise covariance matrix & \multicolumn{3}{l}{ RMSE [deg] } \\
\cline { 2 - 4 } & Ankle joint & Knee joint & Hip joint \\
\hline NBS & 3.17 & 2.41 & 3.18 \\
NBS (Only process noise) & 4.80 & 3.24 & 3.41 \\
NBS (Only observation noise) & 4.71 & 2.57 & 3.22 \\
CNC & 4.88 & 2.54 & 3.24 \\
\hline
\end{tabular}

from NBS (Only observation noise) is much smaller than the result obtained from the optical 3D motion analysis system. The results indicate that the process noise covariance matrix based on the gyroscope output contributed to increased accuracy of the dorsiflexion peak during the swing phase.

In the early stance phase and the end of the swing phase, the ankle joint angle obtained from NBS (Only process noise) is much smaller than the result obtained from the optical 3D motion analysis system, whereas the ankle joint angle obtained from NBS (Only observation noise) is generally consistent with the result obtained using the optical 3D motion analysis system. The results indicate that the observation noise covariance matrix based on the accelerometer and magnetometer output contributed to increased accuracy at the early stance phase and at the end of the swing 
phase. Therefore, the process noise covariance matrix based on the gyroscope output and the observed noise covariance matrix based on the accelerometer and magnetometer output might have contributed to the increased accuracy at different phases.

For knee and hip joint angles, all results show the same tendency. However, NBS (red line) has the smallest RMSE in all results of all three joints. The results show that using both processes of noise covariance matrix based on the gyroscope output and the observed noise covariance matrix based on the accelerometer and magnetometer output might have contributed to increased accuracy. The two noise covariance matrices seem to have influenced one another.

\section{Running experiment}

\section{Participants and experiment conditions}

The nine-axis motion sensors measured lower limb joint angles of the same participants while they were running in place to verify the effectiveness of NBS when continuously capturing data of fast-moving participants. The nine-axis motion sensors were placed in the same positions as those used for the verification experiment. The measurement time was about $100 \mathrm{~s}$. During the experiment, kinematic data were collected using an optical 3D motion analysis system with four nine-axis motion sensors in synchronization. Participants were instructed to run in place in time with a metronome $(150 \mathrm{bpm})$ after maintaining the upright posture for about $5 \mathrm{~s}$. The sampling frequencies of the nine-axis motion sensors and the optical 3D motion analysis system were $100 \mathrm{~Hz}$.

\section{Results}

Table 4 shows parameters $a$ to $f$ for the running.

experiment, which were determined to maximize the log-likelihood in Eq. (22). From the running experiment, different parameters were obtained among the joints. In addition, parameters $a, c$, and $e$ for running measurements tended to be larger than those in the walking measurement. The results indicate that the noise covariance matrices for the running experiment might have had larger values because the process and observation noise can increase if the motion velocity increases.

The estimated joint angles of participant A are presented in Figs. 4, 5, and 6. In each of Figs. 4, 5, and 6 , panels (a) present results obtained over the entire measurement time. Panels (b) present results obtained between $33 \mathrm{~s}$ and $35.5 \mathrm{~s}$ from the start of measurements. In each of Figs. 4, 5, and 6, panels (b) are used for a detailed examination of the results. Black solid curves present results obtained from the optical 3D motion analysis system. Red solid curves present results obtained from NBS. Blue solid curves present results obtained from CNC.

The estimated ankle joint angle using NBS.

Table 4 Adjusting parameters of NBS in the running experiment

\begin{tabular}{|c|c|c|c|c|c|c|}
\hline \multicolumn{7}{|c|}{ (a) Ankle joint } \\
\hline \multirow[t]{2}{*}{ Participant } & \multicolumn{6}{|c|}{ Adjusting parameters } \\
\hline & $a$ & $b$ & c & $d$ & $e$ & $f$ \\
\hline A & 0.1 & 0 & 10 & 0 & 1000 & 0 \\
\hline B & 0.1 & 0 & 10 & 0 & 1000 & 0 \\
\hline C & 0.1 & 0 & 10 & 0 & 1000 & 0 \\
\hline \multicolumn{7}{|c|}{ (b) Knee joint } \\
\hline \multirow[t]{2}{*}{ Participant } & \multicolumn{6}{|c|}{ Adjusting parameters } \\
\hline & $a$ & $b$ & c & $d$ & $e$ & $f$ \\
\hline A & 0.00001 & 0 & 0.1 & 0 & 1 & 0 \\
\hline B & 0.00001 & 0 & 100 & 0 & 100 & 0 \\
\hline C & 0.00001 & 0 & 100 & 0 & 1000 & 0 \\
\hline \multicolumn{7}{|l|}{ (c) Hip joint } \\
\hline \multirow[t]{2}{*}{ Participant } & \multicolumn{6}{|c|}{ Adjusting parameters } \\
\hline & $a$ & $b$ & c & $d$ & $e$ & $f$ \\
\hline A & 0.1 & 0 & 10 & 0 & 1000 & 0 \\
\hline B & 0.001 & 0 & 10 & 0 & 1000 & 0 \\
\hline C & 0.001 & 0 & 10 & 0 & 1000 & 0 \\
\hline
\end{tabular}



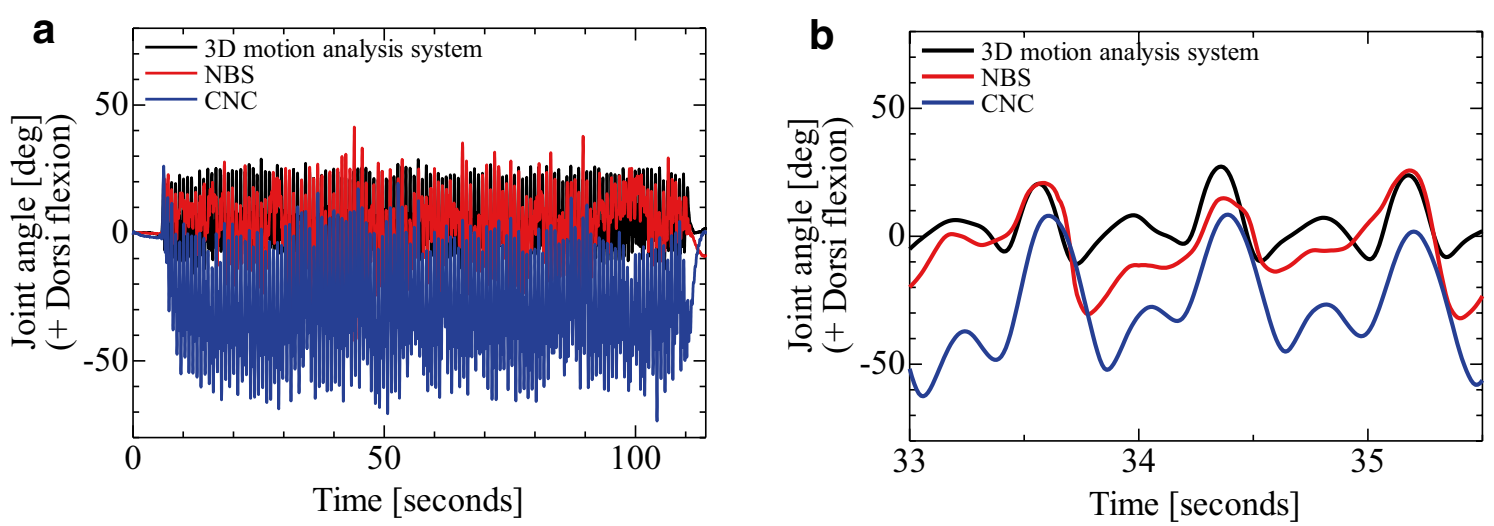

Fig. 4 Results obtained for ankle joint angles (Subject A). a The results over the entire measurement time. $\mathbf{b}$ The results between 33 and $35.5 \mathrm{~s}$.
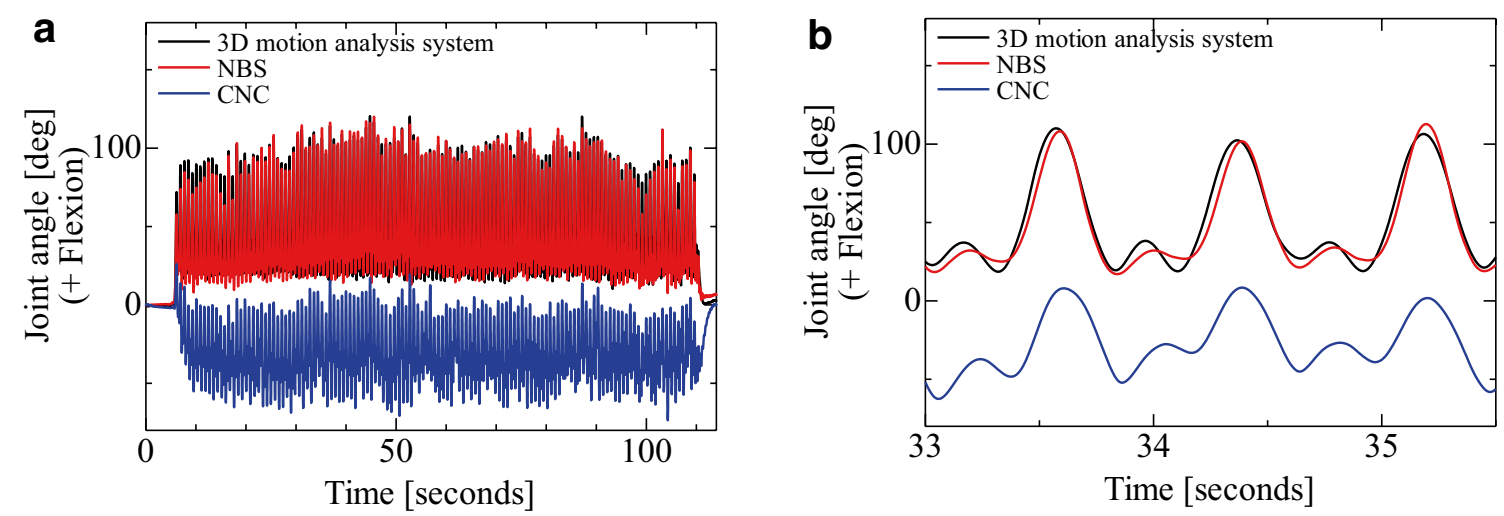

Fig. 5 Results obtained for knee joint angles (Subject A). a The results over the entire measurement time. b The results between 33 and $35.5 \mathrm{~s}$.
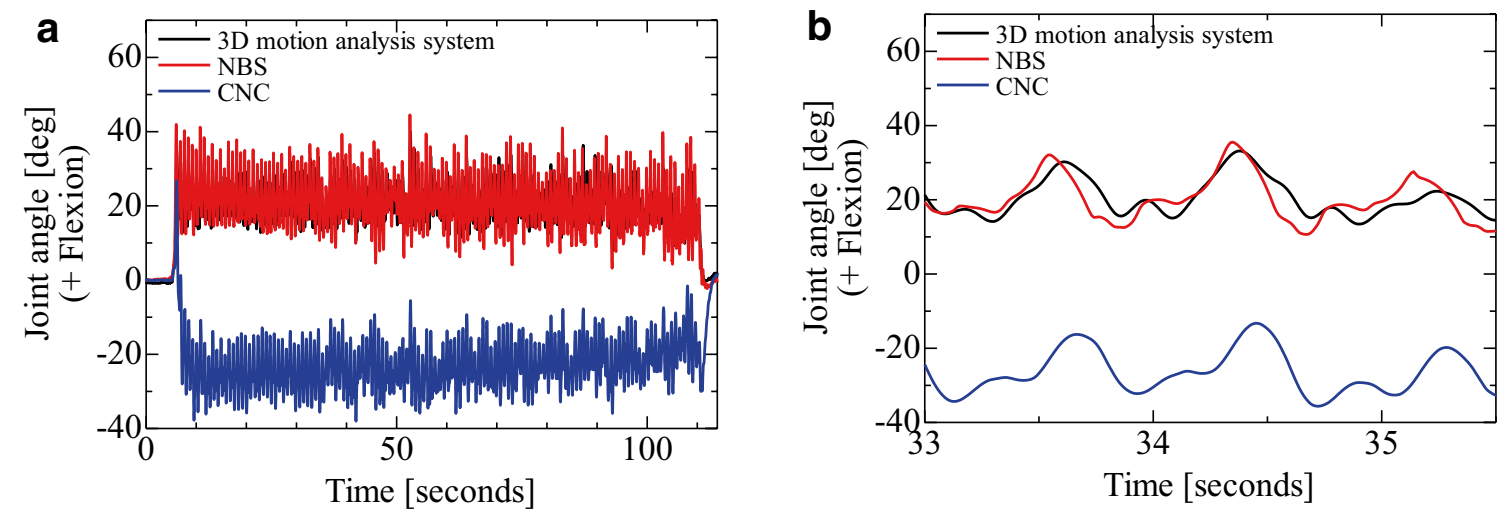

Fig. 6 Results of hip joint angles (Subject A). a The results over the entire measurement time. b The results between 33 and $35.5 \mathrm{~s}$.

in Fig. 4(a) changes periodically between $-25^{\circ}$ and $25^{\circ}$ over the entire measurement time, which is generally consistent with results obtained using the optical 3D motion analysis system. The estimated ankle joint angle using $\mathrm{CNC}$ in Fig. 4(a) changes periodically between $-70^{\circ}$ and $0^{\circ}$ over the entire measurement time. Although 
the waveform of the result obtained using $\mathrm{CNC}$ in Fig. 4(b) is similar to the result obtained using NBS, the result obtained using $\mathrm{CNC}$ is much smaller than that obtained using NBS. Additionally, the waveform of the result obtained using $\mathrm{CNC}$ has a larger dorsiflexion peak than that obtained using NBS at about 33.7, 34.4, and $35.2 \mathrm{~s}$.

The estimated knee joint angle obtained using NBS in Fig. 5(a) changes periodically between $20^{\circ}$ and $110^{\circ}$ over the entire measurement time, which are generally consistent with the results obtained using the optical 3D motion analysis system. Whereas the estimated knee joint angle using CNC in Fig. 5(b) changes periodically between $-60^{\circ}$ and $0^{\circ}$ over the entire measurement time. Although the waveform of the result obtained using CNC in Fig. 5(b) is similar to the result obtained using NBS, the result obtained using $\mathrm{CNC}$ is much smaller than that obtained using NBS. Additionally, the waveform of the result obtained using $\mathrm{CNC}$ has a smaller flexion peak than that obtained using NBS at about 33.6, 34.35, and $35.2 \mathrm{~s}$.

The hip joint angle estimated using NBS in Fig. 6(a) changes periodically between $10^{\circ}$ and $35^{\circ}$ over the entire measurement time, which are generally consistent with results obtained using the optical 3D motion analysis system. The estimated knee joint angle using $\mathrm{CNC}$ in Fig. $6(\mathrm{a})$ changes periodically between $-35^{\circ}$ and $-15^{\circ}$ over the entire measurement time. Although the waveform of the result obtained using CNC in Fig. 6(b) is similar to the result obtained using NBS, the result obtained using $\mathrm{CNC}$ is much smaller than that obtained using NBS. All results obtained for the other two participants showed similar tendencies. The results demonstrated the effectiveness of the extended Kalman filter using NBS.

\section{Conclusions}

For this study, a method for ascertaining the process and observation noise covariance matrices in the extended Kalman filter based on sensor output was constructed to estimate the lower limb joint angles. The lower limb joint angles of the three healthy participants during walking and running were estimated using the method. Results yielded the following conclusions.

1. The joint angles obtained from the extended Kalman filter using the process and observation noise covariance matrices based on sensor output were generally consistent with results obtained using the optical 3D motion analysis system in the verification experiment.

2. In the running motion analysis, the results obtained using noise covariance matrices based on sensor output indicated that the estimated joint angles changed periodically within an appropriate range. The results obtained using the constant noise matrices covariance indicated that the estimated joint angles changed abnormally.

Noise covariance matrices based on sensor output can be effective for accurate pose estimation because noise covariance matrices can be time-variable when continuously capturing human motion with long-term measurements. The proposed method is expected to be useful for estimating motion in sports and healthcare applications.

\section{Acknowledgements}

Not applicable

\section{Authors' contributions}

AS conceived the study and drafted the manuscript. AS and KM carried out all experiments and analyzed the data. YK and SK participated in the research design and sequence alignment. All authors read and approved the final manuscript.

\section{Funding}

This work was supported by JSPS KAKENHI Grant Number JP 20 K19588.

\section{Competing interests}

The authors declare that they have no competing interests.

\section{Author details}

${ }^{1}$ Department of Mechanical Science and Engineering, Kogakuin University, 2665-1 Nakanomachi, Hachioji, Tokyo 192-0015, Japan. ${ }^{2}$ Department of Mechanical Engineering and Robotics, National Institute of Technology (KOSEN), Akita College, 1-1 lijima-Bunkyo-cho, Akita 011-8511, Japan.

Received: 13 May 2020 Accepted: 7 October 2020

Published online: 19 October 2020

\section{References}

1. King K, Yoon SW, Perkins NC, Najaf K (2008) Wireless MEMS inertial sensor system for golf swing dynamics. Sens Actuators, A 141(2):619-630

2. Favre J, Aissaoui R, Jolles BM, Guise JA, Aminian K (2009) Functional calibration procedure for 3D knee joint angle description using inertial sensors. J Biomech 42(14):2330-2335

3. Ameli S, Naghdy F, Stirling D, Naghdy G, Aghmesheh M (2017) Objective clinical gait analysis using inertial sensors and six minute walking test. Pattern Recogn 63:246-257

4. Nüesch C, Roos E, Pagenstert G, Mündermann A (2017) Measuring joint kinematics of treadmill walking and running: Comparison between an inertial sensor based system and a camera-based system. J Biomech 57:32-38

5. Kruk E, Schwab AL, Helm FCT, Veeger HEJ (2018) Getting in shape: Reconstructing three-dimensional long-track speed skating kinematics by comparing several body pose reconstruction techniques. J Biomech 69:103-112

6. Bavan L, Surmacz K, Beard D, Mellon S, Rees J (2019) Adherence monitoring of rehabilitation exercise with inertial sensors: a clinical validation study. Gait Posture 70:211-217

7. Hullfish TJ, Qu F, Stoeckl BD, Gebhard PM, Mauck RL, Baxter JR (2019) Measuring clinically relevant knee motion with a self-calibrated wearable sensor. J Biomech 89(24):105-109

8. Nazarahari M, Noamani A, Ahmadian N, Rouhani H (2019) Sensor-to-body calibration procedure for clinical motion analysis of lower limb using magnetic and inertial measurement units. J Biomech 85(6):224-229

9. Hirose K, Doki H, Koda S, Nagasaku K (2011) Studies of the dynamic analysis and motion measurement of skiing turn using extended Kalman filter. Trans J.jn Soc Mech Eng, Series C 77(774):470-480 ((in Japanese)) 
10. Chardonnens J, Favre J, Cuendet F, Gremion G, Aminian K (2013) A system to measure the kinematics during the entire ski jump sequence using inertial sensors. J Biomech 46(1):56-62

11. Hirose K, Doki H, Kondo A (2012) Studies of orientation measurement in sports using inertial and magnetic field sensors. Jpn Soc Sports Ind 22(2):255-262 ((in Japanese))

12. Saito A, Miyawaki K, Kizawa S, Kobayashi Y (2018) A study of estimating the knee joint angle during walking using the motion sensors (Focusing on the effect of centrifugal acceleration and tangential acceleration). Trans JSME. https://doi.org/10.1299/transjsme.17-00488 (in Japanese)

13. Saito A, Miyawaki K, Komatsu A, Iwami T (2018) A study of sensor position for thigh and lower leg motion sensors during walking (Focusing on the knee sagittal plane angle). Trans JSME. https://doi.org/10.1299/trans jsme.18-00263 (in Japanese)

14. Kondo A, Doki H, Hirose K (2013) A study of calibration method of magnetic field sensor for body motion measurement using inertial sensor. Proc Sports Eng Human Dyn. https://doi.org/10.1299/jsmeshd.2013._2121 (in Japanese)

15. Saito A, Nara Y, Miyawaki K (2019) A study of estimating knee joint angle using motion sensors under conditions of magnetic field variation. Trans JSME. https://doi.org/10.1299/transjsme.19-00061 (in Japanese)

16. Rigatos GG (2010) Extended Kalman and Particle Filtering for sensor fusion in motion control of mobile robots. Math Comput Simul 81(3):590-607

17. Adachi S, Maruta I (2012) Fundamentals of Kalman filter. Tokyo Denki University Press, Tokyo, pp 95-111

18. Ran C, Deng Z (2012) Self-tuning weighted measurement fusion Kalman filtering algorithm. Comput Stat Data Anal 56(6):2112-2128

19. Kamil M, Chobtrong T, Günes E, Haid M (2014) Low-cost object tracking with MEMS sensors, Kalman filtering and simplified two-filter-smoothing. Appl Math Comput 235:323-331

20. Zheng Z, Qiu H, Wang Z, Luo S, Lei Y (2019) Data fusion based multi-rate Kalman filtering with unknown input for on-line estimation of dynamic displacements. Measurement 131:211-218

21. Baerveldt AJ, Klang R (1997) A low-cost and low-weight attitude estimation system for an autonomous helicopter. In: Proceedings of IEEE International Conference on Intelligent Engineering Systems. https://doi. org/10.1109/INES.1997.632450.

22. Mahony RE, Hamel T, Pflimlin JM, (2005) Complementary filter design on the special orthogonal group SO(3). In: Proceedings of the 44th IEEE Conference on Decision and Control, and the European Control Conference 2005. https://doi.org/10.1109/CDC.2005.1582367
23. Euston M, Coote P, Mahony R, Kim J, Hamel T (2008) A complementary filter for attitude estimation of a fixed-wing UAV. In: Proceedings of 2008 IEEE/RSJ International Conference of Intelligent Robots and Systems. https://doi.org/10.1109/IROS.2008.4650766

24. Mahony R, Hamel T, Pflimlin JM (2008) Nonlinear complementary filters on the special orthogonal group. IEEE Trans Autom Control 53(5):1203-1218

25. Sugihara T, Masuya K, Yamamoto M (2013) A complementary filter for high-fidelity attitude estimation based on decoupled linear/nonlinear properties of inertial sensors. J Robot Soc Jpn 31(3):251-262 ((in Japanese))

26. Aoki K (2008) The brand-new technology: Motion analysis system. J Soc Biomech 32(3):167-172 ((in Japanese))

27. Ehara Y (2008) The VICON. J Soc Biomech 32(2):107-111 ((in Japanese))

28. Syam PN, Sheila G, Graham A, Rami A, Weijie W (2010) A method to calculate the centre of the ankle joint: a comparison with the Vicon ${ }^{\circledR}$ Plug-in-Gait model. Clin Biomech 25(6):582-587

29. Stief F, Böhm H, Michel K, Schwirtz A, Döderlein L (2013) Reliability and accuracy in three-dimensional gait analysis: a comparison of two lower body protocols. J Appl Biomech 29:105-111

30. Vaganay J, Aldon MJ, Fournier A (1993) Mobile robot attitude estimation by fusion of inertial data. In: Proceedings of the IEEE International Conference on Robotics and Automation. https://doi.org/10.1109/ROBOT .1993.291995

31. Jurman D, Jankovec M, Kamnik R, Topic M (2007) Calibration and data fusion solution for the miniature attitude and heading reference system. Sens Actuators, A 138(2):411-420

32. Hirose K, Kondo A (2014) Special issues no. 3: measurement technique for ergonomics. Jpn J Ergon 50(4):182-190 ((in Japanese))

33. Miyamoto G, Sonobe M, Shibata K, Hirose K (2019) Development of gait measurement system using IMU sensors. Proc Chugoku and Shikoku Reg Conf . https://doi.org/10.1299/jsmecs.2019.57.1001 ((in Japanese))

34. Ae M, Tang H, Yokoi T (1992) Estimation of inertia properties of the body segments in Japanese athletes. Biomechanism 11:23-33 ((in Japanese))

35. Yamamoto $H$, Yanagida $Y$ (2011) The various patterns of knee angle in the stance phase. Soc Phys Ther Sci 26(2):269-273 ((in Japanese))

\section{Publisher's Note}

Springer Nature remains neutral with regard to jurisdictional claims in published maps and institutional affiliations.

\section{Submit your manuscript to a SpringerOpen ${ }^{\circ}$ journal and benefit from:}

- Convenient online submission

- Rigorous peer review

- Open access: articles freely available online

- High visibility within the field

- Retaining the copyright to your article

Submit your next manuscript at springeropen.com 